\title{
Laws of Gravity and Electrostatics Reduce Elementary Particles to Only Two - Positron and Negatron
}

\author{
Misheck Kirimi*1 \\ Moi University, Kesses, P.O. Box 3900-30100 Eldoret, Kenya
}

\begin{abstract}
I demonstrate that the macrocosmic gravitational interaction between two masses and the microcosmic electrostatic interaction between two charges unify in simple concepts and mathematical laws when electric charge and ordinary mass are interpreted in reciprocal terms. No previous research has ever attempted to unify charge and mass. The difficulty has been lack of an intelligible definition of charge. A three-point paradigm shift solves the problem, giving convincing evidence-for the first time - that positron and negatron are the ultimate elementary particles. That is, matter is pure positive and negative grains of electricity. Paradigm shift \#1: Electron is a moving charge; a charge is static electron - 'one entity two identities'. This implies that ordinary matter contains equal numbers of positive and negative electrons observed in motion as 'electrons' and at rest as 'charges'. In motion, a positron-negatron pair obeys the laws of electrodynamics and annihilates; at rest, the pair obeys the laws of electrostatics and neutralizes. Paradigm shift \#2: Electron mass and electrostatic field are either positive or negative. Opposite masses and fields, rather than indefinable 'charge', make opposite electrons physically different. Paradigm shift \#3: Electric charge and ordinary mass interconvert. Positive charge $\left(e^{+}\right)$and negative charge $\left(e^{-}\right)$neutralize to neutral charge $\left(2 e^{0}\right)$, which is nature's quantum of ordinary mass. Conversely, a quantum of ordinary mass splits to opposite charges e.g. in frictional electrification. The insights systematize the search, identification and classification of 'elementary particles', ending decades of confusion in the 'elementary particle zoo'. They identify a new, stable subatomic particle - a third nucleon.
\end{abstract}

Keywords: Electron, electrostatics, elementary particles, fields, gravity, unification

\section{Introduction}

Newton discovered that masses $M_{1}$ and $M_{2}$ stationed distance $r$ apart attract each other with a force $(F)$ that is directly proportional to the product of the masses $\left(M_{1} M_{2}\right)$ and inversely proportional to the square of the distance $\left(r^{-2}\right)$. Rearranging his equation: $\mathrm{Fr}^{2} / \mathrm{M}_{1} \mathrm{M}_{2}=\mathrm{G}$. About a century later Coulomb discovered that quantities of charge $Q_{1}$ and $Q_{2}$ obey a similar law: $\mathrm{Fr}^{2} / Q_{1} Q_{2}=K$. The two equations have a common mathematical configuration. In addition, the numerators describe the same entity $\left(\mathrm{Fr}^{2}\right)$. However, the products of masses $\left(M_{1} M_{2}\right)$ and charges $\left(Q_{1} Q_{2}\right)$ swop places. As a result, constants $G$ and $K$ are different.

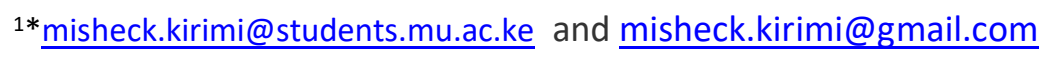


Misheck Kirimi: Matter is Pure Positive and Negative Grains of Electricity

Attempts to explain the similarities and differences between Newton's and Coulomb's laws started soon after Coulomb published his finding ${ }^{1)}$. For over 230 years, however, unification of electric and gravitational phenomena, in general, has eluded "some of the greatest minds in science" ${ }^{2)}$ - including Faraday ${ }^{3)}$, Einstein ${ }^{4)}$ and Wey $^{(5)}$. The long and fruitless search may mean that the phenomena have no natural link; or that a paradigm shift is required to spot the link.

\section{Recent Studies}

Fragmented evidence hints that electrostatics and gravity are different manifestations of a common interaction. For example, Spears derives gravitation constant $(G)$ using only electrostatic parameters and concludes that "gravity is almost certainly an electrostatic phenomenon" ${ }^{6)}$. But his findings remain at the level of mathematical abstraction without giving physical meaning to the numerical relations. Similarly, Greulich demonstrates that "gravitation can be rewritten completely as electrostatics" as long as one assigns to matter (ordinary mass) "a very small gravitational charge density"7). Greulich treats ordinary mass as a composite of infinitesimal quanta of electric charge and finds that 'electric charge density' and 'gravitational charge density' amount to the same thing. However, he does not explain what 'charge densities' mean in physical sense and why gravity is only attractive. Haug, in agreement with Greulich, observes that for Planck masses Newton's formula of gravitation is mathematically exactly the same as Coulomb's formula of electrostatics. His observation suggests that at the most basic level of material structure - "at the very bottom of the rabbit hole" ${ }^{8)}$ - charge and mass have a common fabric. In a related study Aspden infers that gravity is the mutual electrostatic action between "material particles that are uncharged"9). In effect, Aspden equates gravitational mass to 'neutral electric charge'. Consistent with this proposal, Assis demonstrates that units of electrically uncharged particles - which he calls "neutral dipoles" - attracting electrostatically result in gravitation. He concludes that "gravitation is the statistical residual force between groups of neutral dipoles"10). More explicitly, he states that "heavy bodies are composed of oppositely charged particles"11). Beyond his statistical argument Assis does not interpret "neutral dipoles" or "oppositely charged particles" in familiar terms.

The studies cited above point at a common inference that 'unification of electrostatics and gravitation' is essentially the 'unification of charge and mass'. The inference agrees with the fact that charge $\left(Q_{1} Q_{2}\right)$ and mass $\left(M_{1} M_{2}\right)$ are the sole variables that Coulomb's and Newton's laws do not share. In other words, electric charge and ordinary mass are independent physical entities with similar and dissimilar interactive features. The similar features make Coulomb's and Newton's laws analogous. The dissimilar features result in two differences. 1) Coulomb's matter (charge) either attracts or repels but Newton's matter (ordinary mass) only attracts. 2) Coulomb's interaction is much stronger than Newton's interaction. The numerical value of Coulomb's constant over Newton's constant (K/G) shows that $\mathrm{Fr}^{2}$ per interacting charges $\left(\mathrm{Fr}^{2} / \mathrm{Q}_{1} \mathrm{Q}_{2}\right)$ is $1.347 \times 10^{20}$ times greater than $\mathrm{Fr}^{2}$ per interacting masses $\left(\mathrm{Fr}^{2} / \mathrm{M}_{1} \mathrm{M}_{2}\right)$. Hence, correct unification of electrostatics and gravitation is only possible if the natural relation of charge to mass is known. No past study has ever recognised or exploited this fact. Consequently, no one has ever proved that gravity and electricity are different aspects of the same phenomenon ${ }^{12)}$.

\section{Page 2 of 18}


Misheck Kirimi: Matter is Pure Positive and Negative Grains of Electricity

\section{Unification of Charge and Mass}

Physics literature has no significant information on how nature relates charge to mass. The underlying difficulty has been lack of clarity on the physical meaning of charge. Although charge has been recognized, analysed and applied for centuries ${ }^{13}$ ), its fundamental nature remains incoherent ${ }^{14,15)}$ - with no clear formulas linking it to better defined parameters ${ }^{16)}$. This is a problem of great importance and "failure to solve it has restrained development in physics" ${ }^{17}$ ). Is charge so rudimentary that it has no further underlying meaning? Is it so unrelated to the rest of the physical realities that it cannot be interpreted in terms of anything else more comprehensible?

There are scientific leads to the fundamental nature of charge. It is known that a quantity of charge forms the physical bedrock of electrostatic (Coulomb's) interaction whereas a quantity of ordinary mass forms the physical bedrock of gravitational (Newton's) interaction. Therefore, the familiar entity that compares with electric charge is everyday mass. Moreover, physicists have established the two features that distinguish charge from ordinary mass. One, charge is quantized. Millikan proved "very directly" ${ }^{18)}$ that a quantity of charge is an aggregate of individual elementary charges. Similar quantization is not observed in ordinary mass. Two, charge is polarised ${ }^{2}$. It has been known since $1730 \mathrm{~s}^{19}$ that the quanta of electricity (charges) occur in positive and negative types. In contrast, positive-negative symmetry is not observed in ordinary mass. The observation leads to explicit conclusion that charge is the 'polarised matter' and ordinary mass is the 'unpolarised matter'. This conclusion has far reaching implications. Polarised matter exhibits electric properties that include electrostatic interaction; unpolarised matter exhibits mechanical properties that include gravitational interaction. Hence, Coulomb's law describes the interaction between quantities of polarised matter and Newton's law gives a parallel account for unpolarised matter. The inference raises an imperative question on whether nature interconverts the two types of matter.

The law of gravity resolves a gravitational mass in two components: unpolarised inertial mass and unpolarised gravitational field (Fig. 1). Newton discovered that altering either the size or the position of a massive body produces instant mechanical effect in another massive body stationed across space. This entails instant transmission of mechanical energy between physical objects that have no obvious mechanical contact. The simplest interpretation is that a gravitational mass is a single mechanical system comprising a tangible (inertial) mass and an intangible (gravitational) field. This interpretation, as demonstrated below, succeeds to explain a number of observations. It means that gravitational field is no less a mechanical reality than inertial mass. The field is an invisible extension of a gravitational mass beyond the edge of its tangible (inertial) mass. Whereas distance separates inertial masses, there is no distance between gravitational masses. In other words, two gravitational masses are mechanically linked and a change in one instantly affects the other. Hence, Newton's Law of Universal Gravity means that gravitational field mechanically connects every gravitational mass to every other gravitational mass. In this light, what seems like instant action at a distance ${ }^{20)}$ is instant action at no distance. Moreover, a mechanical field that originates in the centre of mass and increases in direct proportion to the quantity

\footnotetext{
2 The terms 'polarised' and 'polarisation' are used here to describe the type of matter that exists in positive and negative types.
} Page 3 of 18 
Misheck Kirimi: Matter is Pure Positive and Negative Grains of Electricity

of mass, and which extends and thins out equally in a three dimension space, has the geometric properties that Einstein interpreted as curved space.

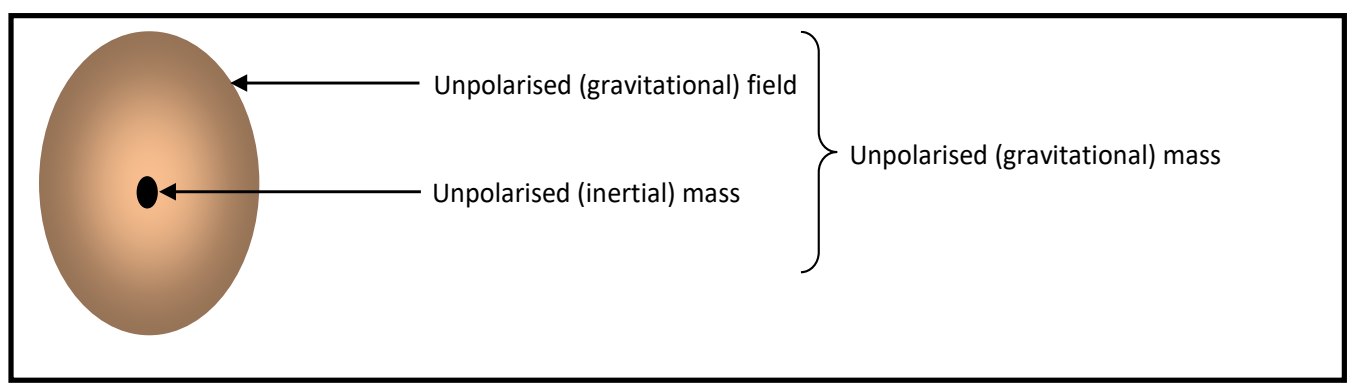

Fig. 1. Newton's gravitational mass comprises unpolarised inertial mass and gravitational field. The field potential at any point is directly proportional to the magnitude of the mass. This shows that inertial mass and gravitational field are inseparable mechanical components of a gravitational mass.

Maxwell had a vision unified "field and substance" ${ }^{21)}$. The vision is realized here for the first time. In the case of gravity, the "field" is gravitational field and "substance" is inertial mass (Fig. 1). Firm theoretical and experimental evidence shows that in the case of a polarized particle (charge) the "field" is the electrostatic field and the "substance" is the electron mass (Fig. 2(a)). Thus the physical components of a charge are the electron mass and the electrostatic field. In this light, the positive-negative charge symmetry is a consequence of positive-negative symmetry in the electron mass and the electrostatic field (Fig. 2(b)). Hence, Coulomb's and Newton's laws relate the same variables - mass, distance and field (detected as force). Differences arise only because Coulomb's "field and substance" are polarised but Newton's are not. This highlights the distinction between gravitational (ordinary) and electric matter.

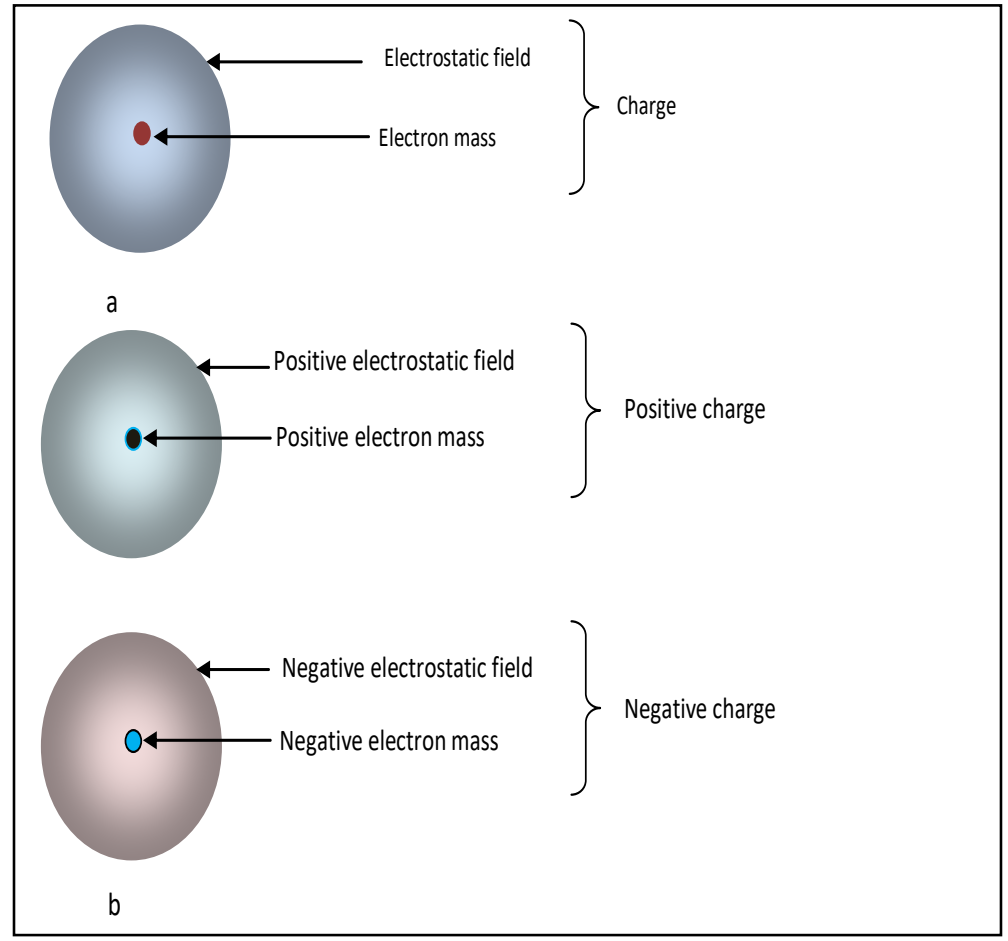

Page 4 of 18
Figs. 2(a) and 2(b). The physical components of a charge (polarised particle) are a blob of electron mass and an electrostatic field around the mass Fig. 2(a). Opposite electron masses and electrostatic field give opposite charges their physical distinctiveness (Fig. 2(b)). 
Misheck Kirimi: Matter is Pure Positive and Negative Grains of Electricity

Test charge experiments demonstrate positive-negative symmetry in electrostatic field. Placed alternately in the fields around opposite charges a test charge deflects in opposite directions ${ }^{22}$. Traditional inference is that field lines face radially outward in positive charge and radially inward in negative charge (Fig. 3). Explicitly, the experiments reveal that electrostatic field - unlike gravitational field - is a polarised phenomenon.
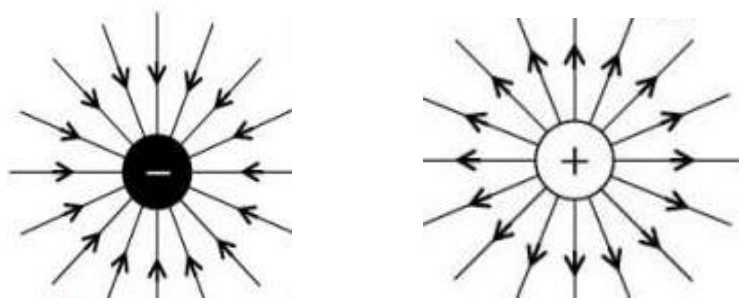

Fig. 3. Test charge experiments detect two types of electrostatic field with opposite behaviours. Traditional interpretation visualizes field lines in a negative charge as facing radially inward and those in a positive charge as facing radially outward.

Dirac, in eq. (1), proves that electron mass $\left(m_{e}\right)$ is either positive $\left(m_{e}{ }^{+}\right)$or negative $\left(m_{e}{ }^{-}\right)$. Schrodinger was the first physicist to observe the fact $^{23}$. However, his observation was downplayed. It was eventually concluded that Dirac's negative mass solution makes only mathematical sense but lacks physical meaning 24, 25). Theorists like Luttinger ${ }^{26)}$, Bondi ${ }^{27)}$ and Price ${ }^{28)}$ give ordinary mass a positive sign. Thus they start with 'known positive ordinary mass' and search for 'unknown negative ordinary mass'. Based on this, it is assumed that electron mass is ordinary and therefore positive. But Dirac's equation does not describe ordinary particles. It describes the electron ${ }^{29)}$ and reveals that electron mass is fundamentally different from ordinary (unpolarised) mass. Dirac and subsequent researchers focused on 'negative energy' and paid no attention to positive-negative electron mass symmetry. This paper rediscovers the fundamental mass symmetry, as expressed in eq. (1), and demonstrates its natural significance.

$$
m_{e}= \pm \sqrt{\frac{E^{2}-p^{2} c^{2}}{c^{4}}}
$$

Dirac's theoretical finding has observational backing. In the cloud chamber photographs Anderson observed a particle (the positron) with properties that counteract those of an ordinary electron (the negatron $)^{30}$. Subjected to uniform force (F) a positron and a negatron, each with mass $m_{e}$, experience acceleration (a) in opposite directions. Newton's second law of motion links these parameters such that 
Misheck Kirimi: Matter is Pure Positive and Negative Grains of Electricity

$\mathrm{F} / \mathrm{m}_{\mathrm{e}}=\mathrm{a}$. When force $(\mathrm{F})$ is fixed - has same magnitude and direction - acceleration (a) can assume opposite signs (occur in opposite directions) only if electron mass $\left(m_{e}\right)$ assumes positive sign $\left(m_{e}{ }^{+}\right)$or negative sign $\left(\mathrm{me}_{\mathrm{e}}^{-}\right)$. In essence, a force that accelerates positron mass to the left will accelerate negatron mass to the right. Anderson's finding, therefore, is an empirical proof that positron and negatron have opposite inertial masses.

Combed, Dirac's equation and test charge experiments simplify electron to a system of polarized electron mass and electrostatic field around the mass (Fig. 1(a)). In terms of its physical components, therefore, electron $(E)$ is can be equated to only electron mass $\left(m_{e}\right)$ and electrostatic field $\left(e_{f}\right)$ :

$$
\mathrm{E}=\mathrm{m}_{\mathrm{e}}+\mathrm{e}_{\mathrm{f}}
$$

Since electron $(E)$ is either positive $\left(E^{+}\right)$or negative $\left(E^{-}\right)$:

$$
\begin{aligned}
& \mathrm{E}^{+}=\mathrm{m}_{\mathrm{e}}^{+}+\mathrm{e}_{\mathrm{f}}^{+} \\
& \mathrm{E}^{-}=\mathrm{m}_{\mathrm{e}}^{-}+\mathrm{e}_{\mathrm{f}}^{-}
\end{aligned}
$$

Eqs. (3) and (4) identify electron mass $\left(m_{e}\right)$ and electrostatic field $\left(e_{f}\right)$ as the physical stuffs that make positron and negatron different. The prevailing view is that positron and negatron are opposite because they carry an independent entity (charge) that exhibits positive-negative properties ${ }^{31)}$. However, sucxh pure charge - detached from electron mass - has never been isolated ${ }^{32}$. Eqs. (3) and (4) remove the need arbitrary charge. Essentially, the electron does not carry any independent charge. Instead, the physical stuffs of which the electron is made constitute charge. This is consistent with the fact that the electron does not lose or gain a charge. If electron lost a charge, two entities would result - pure elementary charge and an electrically neutral electron mass. Neither of the entities has ever been observed.

Unlike the electron, however, particles that actually carry charge are known to both lose and gain an independent physical entity identifiable as a charge. For example, the primary carrier of the positive charge (proton) loses positive charge when it emits a positive electron (positron) in B+ decay. A vital clue that has always been overlooked is that a lost (or gained) elementary charge is always observed as an electron. This fact suggests that electron (E) and elementary charge (e) are different manifestations of the same particle. Electron and elementary charge, however, manifest different empirical behaviours. While opposite electrons annihilate into radiant energy ${ }^{33)}$, opposite charges neutralize into electrically inert matter. The discrepancy is logically explained once electron is recognized as a charge in motion and

\section{Page 6 of 18}


Misheck Kirimi: Matter is Pure Positive and Negative Grains of Electricity

a charge as electron at rest. Thus electron and elementary charge represent one entity with two identities. In motion, a positron-negatron pair obeys the laws of electrodynamics and annihilates. Conversely, at rest the pair obeys the laws of electrostatics and neutralizes.

Electron $(E)$ and the elementary charge (e) are intrinsically the same thing. Therefore, elementary charge (e), like the electron, is the sum of electron mass $\left(m_{e}\right)$ and electrostatic field $\left(e_{f}\right)-e q$. (5). Hence, positive charge $\left(e^{+}\right)$is the sum of positive electron mass $\left(m_{e}^{+}\right)$and electrostatic field $\left(e_{f}^{+}\right)-$eq. (6). Equally, negative charge $\left(e^{-}\right)$is the sum of negative electron mass $\left(m_{e}^{-}\right)$and electrostatic field $\left(e_{f}^{-}\right)$as displayed in eq. (7).

$$
\begin{gathered}
\mathrm{e}=\mathrm{m}_{\mathrm{e}}+\mathrm{e}_{\mathrm{f}} \\
\mathrm{e}^{+}=\mathrm{m}_{\mathrm{e}}^{+}+\mathrm{e}_{\mathrm{f}}^{+} \\
\mathrm{e}^{-}=\mathrm{m}_{\mathrm{e}}^{-}+\mathrm{e}_{\mathrm{f}}^{-}
\end{gathered}
$$

Interpretation of elementary charge as a static electron sheds new light on the composition of the atom. It is known that an atom is electrically neutral because it contains equal numbers of positive and negative charges. The positive-negative charge symmetry is associated with equal numbers of protons and negative electrons ${ }^{34)}$. Treating proton's positive charge is a static positron means that the atom's overall electric neutrality is due to equal numbers of static positive and negative electrons (charges). Hence, hydrogen atoms comprises three subatomic particles: orbital static negatron, nucleus static positron (observed as positive charge) and the electrically neutral part of the proton (Fig. 4).

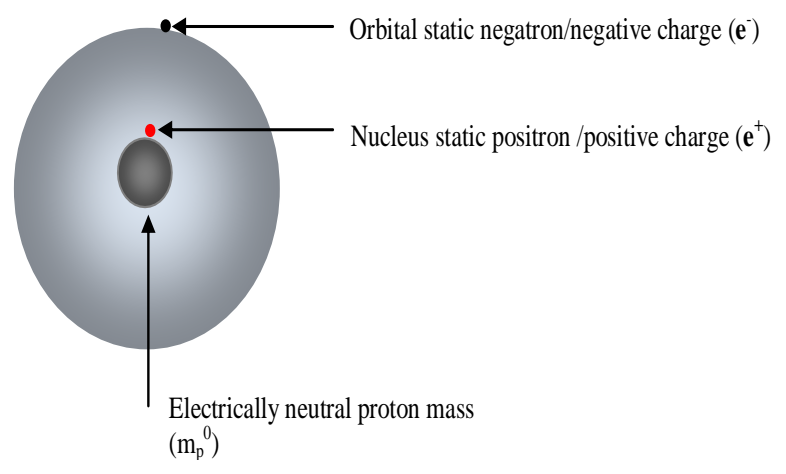

Fig. 4. Recognition of electron as static charge reveals that hydrogen atom comprises three subatomic particles: static negative electron (negative charge), static positive electron (positive charge) and the electrically neutral proton mass. Other atoms are integral multiples of the three basic particles. 
Misheck Kirimi: Matter is Pure Positive and Negative Grains of Electricity

Positive beta $\left(\Omega_{+}\right)$decay directly proves that positive charge is always a static positron. If proton's positive charge is an independent entity, the entity, rather than positron, would be emitted in $ß+$ decay. The fact that positron is emitted without an accompanying negatron precludes the possibility that it arises from electron pair generation. These facts force the inference that a positron pre-exists in the nucleus in static state. Thus ß+ decay converts static positron (positive charge) to moving positron - which is emitted. The initial discovery of the positron in cosmic radiations left a wrong but persisting impression that it is an alien, antimatter particle. But a cumulative body of evidence proves that positron is a universal component of ordinary atoms. For example, in less than two years after Anderson's discovery of the positron from the outer space, Curie and her co-workers discovered the same positron in the isotopes of ordinary matter ${ }^{35)}$. Since then, generation of positrons from ordinary atoms has become a common practice ${ }^{36)}$. Hence, static positrons (positive charges) are no less part of ordinary atoms than the static negatrons (negative charges). Hence, contrary to the view that negative electrons dominate our immediate universe ${ }^{37)}$, available evidence leaves no doubt that ordinary matter contains perfectly equal numbers of positive and negative electrons - observable in motion as 'electrons' and at rest as 'charges'.

Electrons in motion and at rest respectively obey the laws of electrodynamics and electrostatics. In motion, a pair of opposite electrons can simultaneously appear (pair production) or disappear (pair annihilation ${ }^{38,39}$. Parallel electrostatic processes are here named 'charge pair production' and 'charge pair neutralisation'. It is shown here that these processes interconvert ordinary mass and electric charge (Fig. 5). Electrodynamics interconvert electrons and radiant energy; electrostatics interconvert charge and ordinary mass. In charge pair production a pair of opposite charges appears simultaneously from ordinary mass. Charge pair production is familiar and easy to observe but it has never been recognized for what it is. The process is commonly observed in frictional electrification, for example when glass rod is rubbed with silk. Charge pair production splits a quantum of ordinary mass to a pair of opposite charges. In reverse, charge pair neutralisation converts a positive charge $\left(\mathrm{e}^{+}\right)$and a negative charge $\left(\mathrm{e}^{-}\right)$to a neutral charge $\left(2 \mathrm{e}^{0}\right)$ - which is the elementary building block of ordinary mass (Fig. 5).

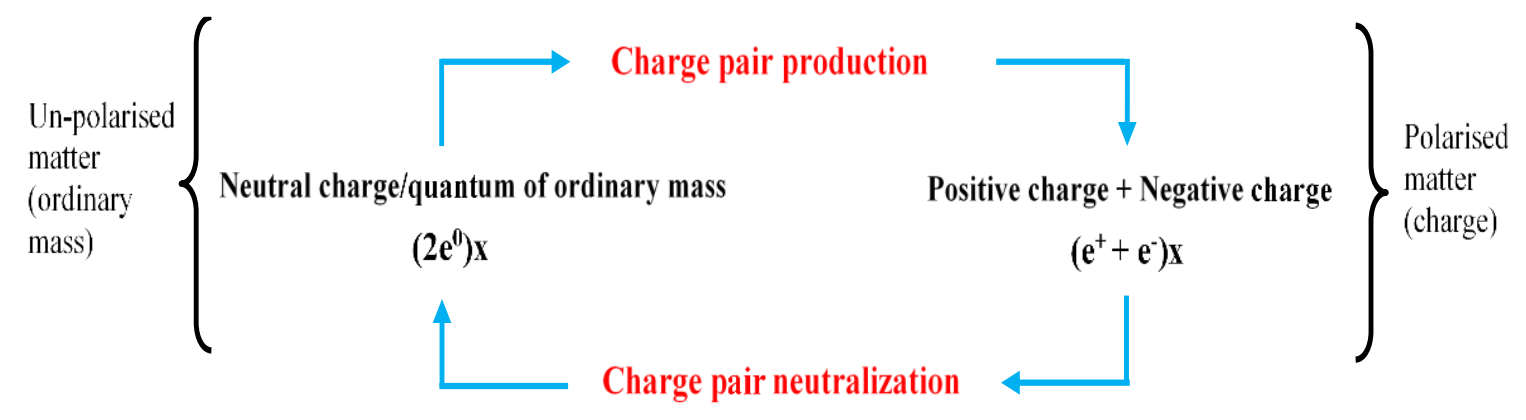

Fig. 5. Processes of 'charge pair production' and 'charge pair neutralisation' explain how nature interconverts unpolarised (ordinary mass) and polarised (electric charge) matter. This reveals, for the first time, that ordinary mass (neutral charge) is internally polarized and quantized.

Page 8 of 18 
Misheck Kirimi: Matter is Pure Positive and Negative Grains of Electricity

In essence, charge pair neutralization transforms the electric features of opposite charges displayed in eq. (6) and eq. (7) to the mechanical features of an ordinary mass particle displayed in eq. (8). That is, the process converts opposite electron masses to a quantum of electrically neutral (unpolarised) mass. Equally, the process converts opposite electrostatic fields to a quantum of electrically neutral (gravitational) field. Thus independent charges exhibit electric properties but opposite charges, coexisting in perfectly equal numbers - either as $2 \mathrm{e}^{0}$ or its integral multiple - and at subatomic proximity, exhibit mechanical properties and are observed as ordinary mass.

$$
\begin{gathered}
\mathrm{e}^{+}=\mathrm{m}_{\mathrm{e}}^{+}+\mathrm{e}_{\mathrm{f}}^{+} \\
+\mathrm{e}^{-}=\mathrm{m}_{\mathrm{e}}^{-}+\mathrm{e}_{\mathrm{f}}^{-} \\
2 \mathrm{e}^{0}=2 \mathrm{~m}_{\mathrm{e}}^{0}+2 \mathrm{e}_{\mathrm{f}}^{0}
\end{gathered}
$$

The full import of charge pair polarization and neutralisation becomes evident when the elements of eq. (8) are tabulated (Table I). The Table shows that positive and negative grains of electricity - occurring in perfectly equal numbers - constitute the foundation of material existence. This simplifies matter to pure quanta of electricity occurring in positive, negative and neutral types.

\begin{tabular}{|c|c|c|c|}
\hline Positive charge $\left(\mathrm{e}^{+}\right)$ & $=$ & Positive electron mass $\left(\mathrm{m}_{\mathrm{e}}^{+}\right)$ & + Positive electrostatic field $\left(\mathrm{e}_{\mathrm{f}}^{+}\right)$ \\
\hline+ & & + & + \\
\hline Negative charge $\left(\mathrm{e}^{-}\right)$ & $=$ & Negative electron mass $\left(\mathrm{m}_{\mathrm{e}}^{-}\right)$ & + Negative electrostatic field $\left(e_{f}^{-}\right)$ \\
\hline$\|$ & & " & $"$ \\
\hline $\begin{array}{l}\text { Neutral charge }\left(2 \mathrm{e}^{0}\right) \text { or quantum } \\
\text { of gravitational mass }\end{array}$ & $=$ & $\begin{array}{l}\text { Neutral electron mass }\left(2 \mathrm{~m}_{\mathrm{e}}^{0}\right) \text { or } \\
\text { quantum of inertial mass }\end{array}$ & $\begin{aligned}+ & \text { Neutral electrostatic field }\left(2 \mathrm{e}_{\mathrm{f}}{ }^{0}\right) \text { or } \\
& \text { quantum of gravitational field }\end{aligned}$ \\
\hline
\end{tabular}

Table I. Natural unification of elementary charge $(e)$, elementary mass $\left(m_{e}\right)$, electrostatic field and gravitational field. The most rudimentary stuff of which the material universe is made are the elementary (electron) mass and the electrostatic field.

\section{Predictions}

Eq. (8) equates e to $m_{e}$ plus $e_{f}$, making conservation of charge the conservation electron mass and electrostatic field. But an electrically neutral particle has an even number of elementary charges - half positive $\left(\mathrm{e}^{+}\right)$and half negative ( $\left.\mathrm{e}^{-}\right)$. Equally, an electrically charged particle has an excess of either of the elementary charges. In unifying elementary charge and the electron mass, eq. (8) predicts that an electrically neutral particle has an even number of electron mass units (EMUs) - half positive $\left(\mathrm{m}_{\mathrm{e}}^{+}\right)$and half negative $\left(\mathrm{m}_{\mathrm{e}}^{-}\right)$. Conversely, a particle that carries an elementary charge must have an odd number of EMUs - with unpaired $\mathrm{m}_{\mathrm{e}}^{+}$or $\mathrm{m}_{\mathrm{e}}^{-}$. The validity or the invalidity of the equation's prediction is testable against existing experimental data. Rounding off the experimental masses of the classical 'elementary particles' ${ }^{\prime 0)}$ to the nearest whole EMU shows that, in agreement with the equation's requirement, particles with even number of EMUs are electrically neutral and the old-numbered ones are electrically charged (Table II). In other words, nature uses 'whole positive and negative electron mass units' as the basic building blocks of mass in in both natural and artificially generated elementary particles. Hence, 
Misheck Kirimi: Matter is Pure Positive and Negative Grains of Electricity

electron mass $\left(m_{e}\right)$ is nature's elementary mass. The decimals in the experimental electron masses may be attributed to such factors as energy-to-mass conversion and experimental/calculation errors.

Table II. Rounding off experimental masses of elementary particles to the nearest whole EMU links conservation of electric charge with conservation of electron mass, showing that electron is nature's elementary unit of charge and mass.

\begin{tabular}{llll}
\hline Multiplet & EMUs and charge states & Even-odd status & Electric charge status \\
\hline \multirow{2}{*}{ Nucleon } & $1837^{+}$ & Odd & Charged \\
\cline { 2 - 4 } & $1840^{\circ}$ & Even & Neutral \\
\hline \multirow{2}{*}{ Pion } & $264^{0}$ & Even & Neutral \\
\cline { 2 - 4 } & $273^{+}$ & Odd & Charged \\
\cline { 2 - 4 } & $965^{+}$ & Odd & Charged \\
\cline { 2 - 4 } & $968^{0}$ & Even & Neutral \\
\hline Eta & $974^{\circ}$ & Even & Neutral \\
\hline Lambda & $1074^{\circ}$ & Even & Neutral \\
\cline { 2 - 4 } & $1077^{+}$ & Odd & Charged \\
\hline Sigma & $2153^{+}$ & Odd & Charged \\
\cline { 2 - 4 } & $2183^{-}$ & Odd & Charged \\
\hline Xi & $2328^{\circ}$ & Even & Neutral \\
\cline { 2 - 4 } & $2343^{-}$ & Odd & Charged \\
\cline { 2 - 4 } & $2573^{-}$ & Odd & Charged \\
\hline
\end{tabular}

Consistent with eq. (8) and Table I, Table II shows that coexisting $\mathrm{me}_{\mathrm{e}}^{+}$and $\mathrm{me}_{\mathrm{e}}{ }^{-}$form $2 \mathrm{~m}_{\mathrm{e}}{ }^{0}-$ which is nature's quantum of inertial mass. Any neutral mass is integral multiple of $2 m_{e}{ }^{0}$ units. This evident in hydrogen atom, provided it is recognized that the units are organized into 'low density outer' and 'high density inner' zones (Fig. 6). In the outer zone, $\mathrm{m}_{\mathrm{e}}{ }^{+}$and $\mathrm{m}_{\mathrm{e}}{ }^{-}$are relatively far apart, resulting in a low density $2 \mathrm{~m}_{\mathrm{e}}{ }^{0}$ units. But the units are tightly packed in the neutral proton mass $\left(m_{p}{ }^{0}\right)$. Hence, the net proton mass usual proton mass less positive elementary mass - form a discrete quantum of ordinary mass. Hence, $m_{p}{ }^{0}$

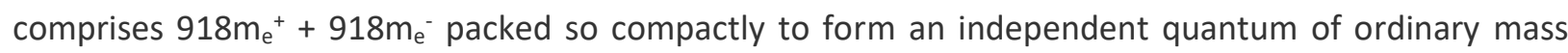
$\left(1836 \mathrm{~m}_{\mathrm{e}}{ }^{0}\right.$ units). Any gravitational mass comprises equal numbers of $2 \mathrm{~m}_{\mathrm{e}}{ }^{0}$ and $\mathrm{m}_{\mathrm{p}}{ }^{0}$ units.

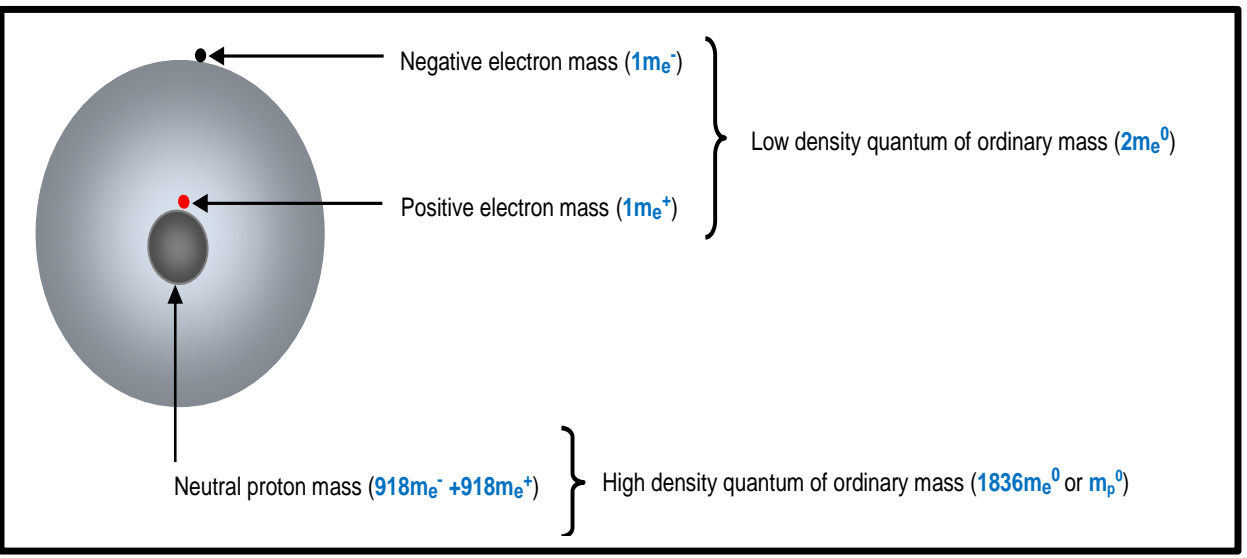

Fig 6. Hydrogen atom comprises low and high density quanta of ordinary mass. The masses of static nuclear positron and orbital negatron constitute the outer quantum. The densely packed $\mathrm{m}_{\mathrm{e}}^{+}$and $\mathrm{m}_{\mathrm{e}}$ - pairs form the high density quantum $\left(m_{p}{ }^{0}\right)$. 
Misheck Kirimi: Matter is Pure Positive and Negative Grains of Electricity

With the exception of nucleons, the subatomic particles listed in Table II are not locatable in the atom. It can be shown, however, that laws that govern proton-neutron inter-conversions apply in all multiplets. First, the mass difference between neutron (1840 EMUs) and proton (1837 EMUs) is 3 EMUs. It is observed here, for the first time, that the 3 EMUs constitute a universal mass phenomenon that differentiates the mass of any subatomic particle and that of its immediate neighbour. As shown in Table III, a series of 3 EMUs neatly links all known particles (bolded) and predicts new ones (un-bolded). Second, proton's charge state has been linked with its odd number of EMUs and neutron's electric neutrality with its even number of EMUs (Table II). Table III extends the nucleonic mass and charge rules to other multiplets. From the table, positive and negative charge states alternate with neutral (zero) states in the interludes. Thus subatomic particles occur in predictable periodicity of EMUs and charge states.

Table III. Extrapolation of electron mass units (EMUs) and electric charge states in known multiplets predicts new elementary particles. A particle differs from its next neighbour by 3 EMUs. Further, particles with even number EMUs are neutral and those with odd number are charged. The highlighted particles have been experimentally detected and an infinite array of other (un-highlighted) particles is predicted.

\begin{tabular}{ll}
\hline Multiplet name & Extended EMUs and charge states (superscripts) in classical multiplets \\
\hline Nucleon & $\ldots 1831^{\circ} \ldots 1834^{0} \ldots 1837^{+} \ldots 1840^{\circ} \ldots 1843^{-} \ldots$ \\
\hline Pion & $\ldots 264^{0} \ldots 267^{-} \ldots 270^{\circ} \ldots 273^{+} \ldots$ \\
\hline Kaon & $\ldots 965^{+} \ldots 968^{0} \ldots 971^{-} \ldots 974^{0} \ldots$ \\
\hline Eta & $\ldots 1071^{-} \ldots 1074^{0} \ldots 1077^{+} \ldots 1080^{\circ} \ldots$ \\
\hline Lambda & $\ldots 2153^{+} \ldots 2156^{\circ} \ldots 2159^{-} \ldots 2162^{\circ} \ldots 2165^{+} \ldots 2168^{\circ} \ldots 2171^{-} \ldots 2174^{0} \ldots 2177^{+} \ldots 2180^{0} \ldots 2183^{-} \ldots$ \\
\hline Sigma & $\ldots 2328^{\circ} \ldots 2331^{-} \ldots 2334^{\circ} \ldots 2337^{+} \ldots 2340^{\circ} \ldots 2343^{\circ} \ldots$ \\
\hline$X i$ & $\ldots 2573^{-} \ldots 2576^{0} \ldots 2579^{+} \ldots$ \\
\hline
\end{tabular}

Transmutation of neutron $\left(\mathrm{N}^{0}\right)$ to proton $\left(\mathrm{P}^{+}\right)$highlights the natural significance of 3 EMUs in nuclear reactions. This is currently expressed as $\mathrm{N}^{0} \rightarrow \mathrm{P}^{+}+\mathrm{e}^{-}+\mathrm{v}_{\mathrm{e}}$, where $\mathrm{e}^{-}$is the negatron and $\mathrm{v}_{\mathrm{e}}$ is the electron antineutrino. The transmutation increases the original atomic number by one, resulting in a new atom. From section 3 of this article, however, proton's positive charge is a static positive electron. Therefore, the traditional proton $\left(\mathrm{P}^{+}\right)$comprises a neutral proton part $\left(\mathrm{P}^{0}\right)$ and a positron $\left(\mathrm{e}^{+}\right)$. That is, $\mathrm{P}^{+}=\left(\mathrm{P}^{0}+\mathrm{e}^{+}\right)$. Since the neutron $\left(\mathrm{N}^{0}\right)$ looses 3 EMUs to become a proton $\left(\mathrm{P}^{+}\right)$, the 3 EMUs and $\mathrm{e}^{-}+\mathrm{v}_{\mathrm{e}}$ mean the same thing. The liberated negatron ( $\left.e^{-}\right)$accounts for $1 \mathrm{EMU}$ and the $v_{e}$ for the remaining 2 EMUs. But eq. (8) identifies a natural unit of matter with 2 EMUs as the neutral electric charge $\left(2 \mathrm{e}^{0}\right)$. In this light, Pauli's neutrino is the neutral electric charge $\left(2 \mathrm{e}^{0}\right)$. Hence, transmutation of neutron to proton can be rewritten as: $\mathrm{N}^{0} \rightarrow\left(\mathrm{P}^{0}+\mathrm{e}^{+}\right)+\mathrm{e}^{-}+2 \mathrm{e}^{0}$. Or, in terms of EMUs as: $1840 \mathrm{me}_{\mathrm{e}}^{0} \rightarrow\left(1836 \mathrm{~m}_{\mathrm{e}}{ }^{0}+1 \mathrm{~m}_{\mathrm{e}}^{+}\right)+1 \mathrm{~m}_{\mathrm{e}}^{-}+2 \mathrm{me}^{0}$.

It is inferred that $\beta+$ decay converts proton to neutron ${ }^{41)}$ - emitting positron $\left(\mathrm{e}^{+}\right)$and electron neutrino $\left(v_{\mathrm{e}}\right)$ in the process. The inference is based on the fact that the atomic number of the original atom decreases by one. The reaction is expressed as: $\mathrm{P}+\rightarrow \mathrm{N}^{0}+\mathrm{e}^{+}+\mathrm{v}_{\mathrm{e}}$. But conversion of proton to three new particles with combined mass greater than its own mass would violate the law of mass-energy conservation. To solve the problem, it was once thought that the neutrino has zero rest mass. Later 
Misheck Kirimi: Matter is Pure Positive and Negative Grains of Electricity

experiments showed that the neutrino has rest mass ${ }^{42}$. Even if the masses of positron and neutrino were discounted, however, the neutron mass would still be greater than the mass of the original proton. The direct implication is that the particle named 'neutron' in ß+ decay is certainly not Chadwick's neutron. Based on these observations, a new subatomic particle is recognized here, for the first time, and named the nairotron. The nairotron is a stable, electrically neutral nucleon with a mass of 1834 EMUs. It is lighter than either the neutron or the proton. Whereas the neutron can undergo negative beta decay, the nairotron can decay - if it decays at all - via the gamma route (see Table IV). If the nairotron is denoted $\mathrm{n}^{0}$, then it can be shown that it is produced when the proton emits a neutrino (neutral charge) and a positron: $\left(\mathrm{P}^{0}+\mathrm{e}^{+}\right) \rightarrow \mathrm{n}^{0}+\mathrm{e}^{+}+2 \mathrm{e}^{0}$. In terms of the EMUs, conversion of proton to nairotron proceeds as follows: $1837 \mathrm{me}_{\mathrm{e}}^{+} \rightarrow 1834 \mathrm{~m}_{\mathrm{e}}^{0}+1 \mathrm{~m}_{\mathrm{e}}^{+}+2 \mathrm{me}^{0}$.

Table IV. Nucleon multiple reveals predictable patterns in nuclear reactions. Any reaction liberates a neutral charge $\left(2 \mathrm{e}^{0}\right)-$ or $^{\circ}$ neutrino - along with one of the following: $\mathrm{e}^{+}, \mathrm{e}^{-}$or both $\mathrm{e}^{+}$and $\mathrm{e}^{-}$. The $2 \mathrm{e}^{0}$ is invariably emitted. As a rule, nature excludes $\mathrm{e}^{-}$ in the nuclei and $\mathrm{e}^{+}$in the orbits. A pair of opposite charges that violating the rule is ejected as gamma radiation (cycle 1). A negative charge violating the rule is emitted as $\beta^{-}$particle (cycle 2 ); and a positive charge as $\beta^{+}$particle (cycle 4). When concurrently liberated $\mathrm{e}^{+}$and $\mathrm{e}^{-}$do not violate the rule, there no emission. Instead, internal adjustments results in a new atom (cycle 3). The patterns identify a new nucleon, the Nairotron, which has previously been mistaken for neutron. The ß-decay routes are highlighted in pink; the $\aleph^{+}$decay routes in grey; and the $\gamma$ decay routes in blue. The patterns systematize the search, identification and classification of 'elementary' particles.

\begin{tabular}{|c|c|c|c|c|}
\hline $\begin{array}{lll}\begin{array}{l}\text { Particle's EMUs \& } \\
\text { charge }\end{array} & & \\
\end{array}$ & Decay route & Liberated charge & $\begin{array}{l}\text { Non neutrino } \\
\text { emission }\end{array}$ & $\begin{array}{l}\text { Repeating } \\
\text { cycles }\end{array}$ \\
\hline 个 $\ldots$ & $\ldots$ & $\ldots$ & ... & $\ldots \uparrow$ \\
\hline Predicted $1846^{\circ}$ & $1846 \mathrm{e}^{0} \rightarrow 1843 \mathrm{e}^{-}+1 \mathrm{e}^{+}+2 \mathrm{e}^{0}$ & Positive \& negative & $\beta^{+}+\beta^{-}(\gamma-$ rays $)$ & 1 \\
\hline Predicted $1843^{-}$ & $1843 \mathrm{e}^{-} \rightarrow 1840 \mathrm{e}^{0}+1 \mathrm{e}^{-}+2 \mathrm{e}^{0}$ & Negative & $\beta^{-}$ & 2 \\
\hline Neutron $1840^{\circ}$ & $1840 \mathrm{e}^{0} \rightarrow 1837 \mathrm{e}^{+}+1 \mathrm{e}^{-}+2 \mathrm{e}^{0}$ & Negative & None & 3 \\
\hline Proton $1837^{+}$ & $1837 \mathrm{e}^{+} \rightarrow 1834 \mathrm{e}^{0}+1 \mathrm{e}^{+}+2 \mathrm{e}^{0}$ & Positive & $\beta^{+}$ & 4 \\
\hline Nairotron $1834^{\circ}$ & $1834 \mathrm{e}^{0} \rightarrow 1831 \mathrm{e}^{-}+1 \mathrm{e}^{+}+2 \mathrm{e}^{0}$ & Positive \& negative & $\beta^{+}+\beta^{-}(\gamma-$ rays $)$ & 1 \\
\hline Predicted $1831^{-}$ & $1831 e^{-} \rightarrow 1828 e^{0}+1 e^{-}+2 e^{0}$ & Negative & $\beta^{-}$ & 2 \\
\hline Predicted $1828^{\circ}$ & $1828 \mathrm{e}^{0} \rightarrow 1825 \mathrm{e}^{+}+1 \mathrm{e}^{-}+2 \mathrm{e}^{0}$ & Negative & None & 3 \\
\hline Predicted $1825^{+}$ & $1825 \mathrm{e}^{+} \rightarrow 1822 \mathrm{e}^{0}+1 \mathrm{e}^{+}+2 \mathrm{e}^{0}$ & Positive & $\beta^{+}$ & 4 \\
\hline Predicted $1822^{0}$ & $1822 \mathrm{e}^{0} \rightarrow 1819 \mathrm{e}^{-}+1 \mathrm{e}^{+}+2 \mathrm{e}^{0}$ & Positive \& negative & $\beta^{+}+\beta^{-}(\gamma-$ rays $)$ & 1 \\
\hline Predicted 1819- & $1819 \mathrm{e}^{-} \rightarrow 1816 \mathrm{e}^{0}+1 \mathrm{e}^{-}+2 \mathrm{e}^{0}$ & Negative & $\beta^{-}$ & 2 \\
\hline Predicted $1816^{\circ}$ & $1816 \mathrm{e}^{0} \rightarrow 1813 \mathrm{e}^{+}+1 \mathrm{e}^{-}+2 \mathrm{e}^{0}$ & Negative & None & 3 \\
\hline Predicted $1813^{+}$ & $1813 \mathrm{e}^{+} \rightarrow 1810 \mathrm{e}^{0}+1 \mathrm{e}^{+}+2 \mathrm{e}^{0}$ & Positive & $\beta^{+}$ & 4 \\
\hline$\downarrow \ldots$ & $\ldots$ & $\ldots$ & $\ldots$ & $\ldots \downarrow$ \\
\hline
\end{tabular}

\section{Unification of Electrostatics and Gravity}

Table I equated ordinary mass to neutral electric charge - a composite of equal numbers of positive and negative charges. Therefore, both Coulomb's and Newton's laws can be expressed in terms of charges. In other words, gravitation can be rewritten as the electrostatic attraction between opposite halves of masses $M_{1}$ and $M_{2}$ : 
Misheck Kirimi: Matter is Pure Positive and Negative Grains of Electricity

$$
\frac{1}{2} M_{1}^{-} \times \frac{1}{2} M_{2}^{+}=\frac{1}{4} M_{1} M_{2}^{-}
$$

Unlike charges - as well as unlike magnetic poles - attract. Eq. (9) extends this rule to ordinary mass, such that 'unlike halves' of 'like masses' attract (Fig. 7). Newton's law shows that unequal gravitational masses, such as the earth and the moon, attract with equal but opposite forces. This suggests that ordinary mass has an internal symmetry. The symmetry is currently explained in terms of 'active' and 'passive' gravitational masses' ${ }^{\prime 33}$. Active and passive gravitational masses are, respectively, defined as the source and the sink of gravitational field lines ${ }^{44}$. A gravitational mass is both the source and the sink of gravitational field lines - half 'active' and half 'passive'. Eq. (9) and Fig. 7 show that the active-passive mass symmetry as the positive-negative elementary mass symmetry within an electrically neutral mass.

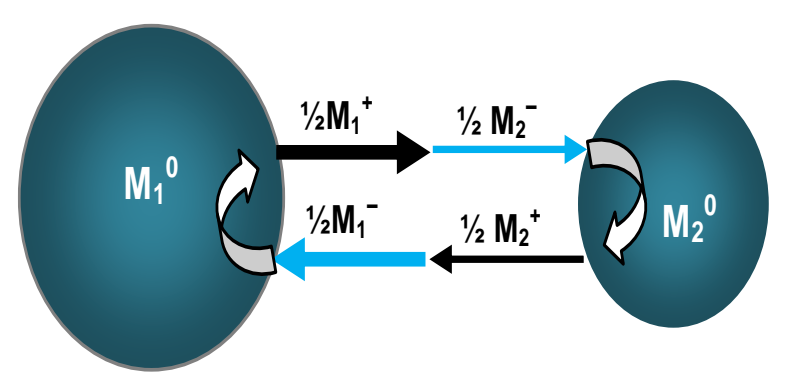

Fig. 7. Gravitational attraction is the electrostatic attraction between unlike halves of like masses. Resolving Newton's mass $\left(M^{0}\right)$ into $1 / 2 M^{+}$and $1 / 2 M^{-}$explains why unequal gravitational masses $M_{1}{ }^{0}$ and $M_{2}{ }^{0}$ attract with equal but opposite forces. Each mass contributes to force in either direction.

The product of opposite masses $\left(1 / 4 \mathrm{M}_{1} \mathrm{M}_{2}^{-}\right)$and the product of opposite charges $\mathrm{Q}_{1} \mathrm{Q}_{2}{ }^{-}$represent the same physical reality - interacting quantities of opposite (polarised) matter or charges. However, polarization alone does not reveal the ultimate equivalence of the respective interactions. This is because Coulomb's and Newton's equations measure the quantities of charge in artificial units. For instance, nature recognizes quantity $Q$ of charge as an aggregate of individual e units but Coulomb's equation recognizes it as an aggregate of individual artificial units - the coulombs (C). In nature, e is one (1) - a natural unit but in Coulomb's equation it is assigned an artificial value of $1.604 \times 10^{-19} \mathrm{C}$. Equally, nature recognizes quantity $M$ of mass as an aggregate of equal numbers of $2 m_{e}{ }^{0}$ and $m_{p}{ }^{0}$ units (Fig. 6). But Newton's equation recognizes a quantity of mass as an aggregate of individual artificial units - the kilograms. To prove that $\mathrm{Q}_{1} \mathrm{Q}_{2}{ }^{-}$and $1 / 4 \mathrm{M}_{1} \mathrm{M}_{2}{ }^{-}$represent the same physical entity, and to express the respective equations in reciprocal terms, both charge and mass must be expressed in natural units. This entails quantization of charge and ordinary mass; or counting matter in terms of elementary charges.

Millikan showed that quantity $Q$ of charge is an integral multiple of the elementary charge (e). Therefore, any $Q_{1} Q_{2}$ is an aggregate of $e^{2}$ units. Hence, quantized $Q_{1} Q_{2}$ is $Q_{1} Q_{2} e^{2}$. Quantity $1 / 4 M_{1} M_{2}$ of mass can be quantized in similar manner. As illustrated in Fig. 6 and demonstrated in part III of this paper, gravitational 


\section{Misheck Kirimi: Matter is Pure Positive and Negative Grains of Electricity}

mass is organized into high density $\left(m_{p}{ }^{0}\right)$ and the low density $\left(2 m_{e}{ }^{0}\right)$ quanta within the atom. Polarising the ordinary mass quanta:

$\mathrm{m}_{\mathrm{p}}^{0}=\frac{1}{2} \mathrm{~m}_{\mathrm{p}}^{+}+\frac{1}{2} \mathrm{~m}_{\mathrm{p}}^{-}$and $2 \mathrm{~m}_{\mathrm{e}}^{0}=\mathrm{m}_{\mathrm{e}}^{+}+\mathrm{m}_{\mathrm{e}}^{-}$

Hence, the natural (smallest) unit of $1 / 4 \mathrm{M}_{1} \mathrm{M}_{2}$ - is:

$\frac{1}{2} \mathrm{~m}_{\mathrm{p}}^{+} \times \frac{1}{2}\left(2 \mathrm{~m}_{\mathrm{e}}\right)^{-}=\frac{1}{2} \mathrm{~m}_{\mathrm{p}} \mathrm{m}_{\mathrm{e}}$

The total number of $1 / 2 m_{p} m_{e}$ units in $1 / 4 M_{1} M_{2}$ is:

$\frac{1}{2} m_{p} m_{e} \times \frac{1}{4} M_{1} M_{2}=\frac{1}{8} M_{1} M_{2} m_{p} m_{e}$

Expressed in natural units, $Q_{1} Q_{2}$ becomes $Q_{1} Q_{2} e^{2}$ and $1 / 4 M_{1} M_{2}$ becomes ${ }^{1} /{ }_{8} M_{1} M_{2} m_{p} m_{e}$. In these forms, the two types of matter that make electrostatics and gravitation different are standardized - polarised and quantized. This opens a way to standardise (unify) Coulomb's and Newton's equations. Coulomb showed that $\mathrm{Fr}^{2} / \mathrm{Q}_{1} \mathrm{Q}_{2}$ is constant $\mathrm{K}$, and Newton showed that $\mathrm{Fr}^{2} / \mathrm{M}_{1} \mathrm{M}_{2}$ is constant $\mathrm{G}$. Matter in the traditional Coulomb's equation $\left(Q_{1} Q_{2}\right)$ is polarised but not quantized. On the other hand, matter in the traditional Newton's equation $\left(\mathrm{M}_{1} \mathrm{M}_{2}\right)$ is neither polarised nor quantized. Polarised and quantized Coulomb's matter (charge) changes his equation to $\mathrm{Fr}^{2} / \mathrm{Q}_{1} \mathrm{Q}_{2} \mathrm{e}^{2}=\mathrm{T}_{\mathrm{c}}$. Equally, polarizing and quantizing Newton's matter (mass) changes his equation to $8 \mathrm{Fr}^{2} / \mathrm{M}_{1} \mathrm{M}_{2} \mathrm{~m}_{\mathrm{p}} \mathrm{m}_{\mathrm{e}}=\mathrm{T}_{\mathrm{n}}$. Calculations based on CODATA values of physical constants ${ }^{45)}$ show that $T_{c}$ equals $3.506 \times 10^{47} \mathrm{NM}^{2} / \mathrm{C}^{4}$ and $T_{n}$ equals $3.506 \times 10^{47} \mathrm{NM}^{2} / \mathrm{kg}^{4}$. The perfect equality $T_{c}$ to $T_{n}$ proves that eq. (8) correctly unifies electric charge and ordinary mass. Equating $T_{c}$ to $T_{n}$ :

$$
\frac{8 \mathrm{Fr}^{2}}{\mathrm{M}_{1} \mathrm{M}_{2} \mathrm{~m}_{\mathrm{p}} \mathrm{m}_{\mathrm{e}}}=\frac{\mathrm{Fr}^{2}}{\mathrm{Q}_{1} \mathrm{Q}_{2} \mathrm{e}^{2}}
$$

Inserting Newton's and Coulomb's constants in eq. (10): 
Misheck Kirimi: Matter is Pure Positive and Negative Grains of Electricity

$$
\frac{8 G}{m_{p} m_{e}}=\frac{K}{e^{2}} .
$$

Eq. (11) show how nature relates the fundamental gravitation-mass constants to the fundamental electrostatic-charge constants. The equation is not only numerical balanced but also conceptually symmetrical. It shows that Coulomb's and Newton's equations can be written under a common constant $(\mathrm{T})$, such that $\mathrm{Te}^{2}=\mathrm{K}$, and $\mathrm{Tm}_{\mathrm{p}} \mathrm{m}_{\mathrm{e}} / 8=\mathrm{G}$. Hence, Newton's equation can be rewritten as:

$$
\mathrm{F}=\frac{\mathrm{TM}_{1} \mathrm{M}_{2} \mathrm{~m}_{\mathrm{p}} \mathrm{m}_{\mathrm{e}}}{8 \mathrm{r}^{2}}
$$

Equally, Coulomb's equation can be written as:

$$
\mathrm{F}=\frac{\mathrm{TQ}_{1} \mathrm{Q}_{2} \mathrm{e}^{2}}{\mathrm{r}^{2}}
$$

Newton's law of gravitation and Coulomb's law of electrostatics have been unified into a more general law, which can be stated as follows: Two quantities of electric charge separated by distance r experience a force $(F)$ that is directly proportional to their product and inversely proportional to the square of the distance $\left(r^{-2}\right)$. In practice, the quantities of charge in Newton's equation are neither polarised nor quantized. On the other hand, the quantities of charge in Coulomb's equation are polarised but not quantized. Only when the quantities of charge in both equations are standardized - polarised and quantized - is the underlying harmony observed as in eq. (11).

\section{Conclusion}

This paper has presented firm evidence that positron and negatron are the ultimate elementary blocks of matter. The discovery could not have been made earlier due to lack of clarity on the fundamental nature of electric charge. A three-point paradigm shift has solved the problem, unifying charge and mass and opening new vistas in physics.

One, the paper has demonstrated that an electron is a moving charge and a charge is a static electron. Electron and elementary charge have previously been regarded as two different entities, with the electron 'carrying' the charge. The confusion led to the inference that negative electrons dominate our immediate universe and that positive electrons are fringe particles. While theories - and particularly 
Misheck Kirimi: Matter is Pure Positive and Negative Grains of Electricity

Dirac's - demand positive-negative electron symmetry in nature, lack of such symmetry has been enigmatic. The new insight shows that our immediate universe has equal numbers of positive and negative electrons - moving and static. In this light, the atom's overall electric neutrality is due to equal numbers of static positive and negative electrons.

Two, the paper has proved that a grain of electricity is not an ordinary particle. Its contents, the electron mass and the electrostatic field, are either positive or negative. The current view is that positron and negatron have the same type of mass but opposite types of electric charge - though 'electric charge' in existing theories is ambiguous. The new insight shows that polarised stuffs, rather than the incoherent 'charge', give opposite electrons physical distinction. Firm theoretical and experimental proof has been presented to show that electron mass and electrostatic fields are either positive or negative.

Three, the paper has shown that electric charge and ordinary mass interconvert. Whereas opposite charges in motion (electrons) annihilate to energy, opposite electrons at rest (charges) neutralize to a quantum of ordinary mass. Conversely, a quantum of ordinary mass splits into a pair of opposite charges. The two processes have been named charge pair production and charge pair neutralisation. It has been shown that charge pair production - the electrostatic equivalence of 'electron pair production' in electrodynamics - splits a quantum of ordinary mass to a pair of opposite charges. In reverse, charge pair neutralisation - the electrostatic equivalence of electron pair annihilation in electrodynamics - converts a pair of opposite charges to a quantum of ordinary mass. That is, positive charge $(e+)$ and negative charge (e-) convert to neutral charge $\left(2 \mathrm{e}^{0}\right)$ - which is nature's elementary block of ordinary mass.

Two vital equations have been derived from the new paradigm: Eq. (8) and Eq. (12). The implications of Eq. (8) have been exhaustively discussed. The equation unifies ordinary mass and electric charge. Among other things, it reveals that electron mass is the natural elementary mass of which atoms and 'elementary' particles are made. The Equation has made it possible to establish previously unknown patterns in nuclear reactions. This has led to identification of a new subatomic particle - a third, stable nucleon. The new particle has been named the Nairotron. In addition, the Equation predicts an infinite array of 'elementary' particles, specifying their mass and electric charge status. On the other hand, Eq. (12) established the long-sought link between gravity and electricity. Broadly, two quantities of electric charge separated by distance $r$ experience a force $(F)$ that is directly proportional to their product and inversely proportional to the square of the distance $\left(r^{-2}\right)$. However, quantities of charge in Newton's equation are neither polarised nor quantized. In contrast, quantities of charge in Coulomb's equation are polarised but not quantized. Standardizing - polarizing and quantizing - quantities of charge in both equations reveals underlying harmony. Unification of gravity and electricity provides a theoretical blueprint for possible manipulation of gravity. Equally, the observed patterns in nuclear reactions pave way to systematic search, identification and classification of 'elementary' particles.

\section{Competing Interests}

The author declared that he has no competing interests. 
Misheck Kirimi: Matter is Pure Positive and Negative Grains of Electricity

\section{References}

1. A. K. T. Assis, Deriving Gravitation from Electromagnetism, Can. J. Phys. 70, 330 - 340 (1992).

2. M. A. El-Lakany, Unification of Gravity and Electromagnetism, Journal of Physical Science and Application 73 15-24 (2017).

3. M. Faraday, On the Possible Relation of Gravity to Electricity, Philosophical Transactions, 141, pp. 1-6. (1851).

4. D. Gross, Einstein and the Search for Unification, Current Science 89, No. 12 (2005).

5. H.Weyl, Electricity and Gravitation, Nature 106, 800-802 (1921).

6. M. F. Spears, An Electrostatic Solution for the Gravity Force and the Value of G, Galilean Electrodynamics, 21, No. 2, pp. 23-32 (2010).

7. K. O. Greulich, A Surprisingly Close Relationship between Gravitation and Electrostatic Interactions, Fritz Lipmann Institute, Beutenbergstr. 11 D 07745 Jena, http://www.flileibniz.de/www_kog/(accessed 28 April 2018).

8. E. G. Haug, Unification of Gravity and Electromagnetism, GravityElectroMagnetism - A Probability Interpretation of Gravity, Norwegian University of Life Science (2016).

9. H. Aspden, Can Gravity Be an Electrostatic Force? Aspden Research Papers, No. 3, (2005).

10. A.K.T. Assis, Advanced Electromagnetism - Foundations, Theory and Application, World Scientific, Singapore, pp. 314-331 (1995).

11. A.K.T. Assis, Deriving Gravitation from Electromagnetism, Can. J. Phys. 70, 330 - 340 (1992).

12. R. Feynman, The Character of Physical Laws, The MIT Press, pp. 30-31(1967).

13. D.H.D. Roller, The Development of the Concept of Electric Charge: Electricity from the Greeks to Coulomb, Cambridge, MA: Harvard University Press. p. 1 (1954).

14. J. N. Mahdi, On the Nature of Electric Charge, Int. J. Phys. Sci. 9(4), pp. 54-60, (2014).

15. V. A. Etkin, Modified Coulomb Law, World Scientific News 87 163-174, (2017).

16. T. Zhang, Electric Charge as a Form of Imaginary Energy, Progress in Physics, 2, pp. 79-83 (2008).

17. L.G. Kreidik and G.P. Shpenkov, Atomic Structure of Matter-Space, Alternative Picture of the World, 1-3, Bydgoszcz, (1996).

18. R. A. Millikan, On the Elementary Electrical Charge and the Avogadro Constant, Phys Rev.2.109 (1913).

19. E. A. Whittaker, History of the Theories of Aether and Electricity, P.1. The Classical Theories. London, (1951).

20. L. J. Wang, Unification of Gravitational and Electromagnetic Forces, J Phys Chem Biophys, (2018).

21. F. Wilczek, Unification of Force and Substance. Phil. Trans. R. Soc. A 374: 20150257 (2016).

22. E.M. Purcell and D. J. Morin, Electricity and Magnetism (3rd Ed.) Harvard University, Cambridge University Press, New York pp. 5-7 (2013).

23. F. Winterberg, Teichmüller Space Interpretation of Quantum Mechanics, Gauss Press, Reno, Nevada, Zf. Naturforsch 58a, 231 (2003). 
Misheck Kirimi: Matter is Pure Positive and Negative Grains of Electricity

24. G. N. Golden, Avoiding Negative Probabilities in Quantum Mechanics, Journal of Modern Physics, 4, 1066-1074 (2013).

25. G. B. Stephen, and S. Ariel, The Making of Twentieth Century Science, How Theories Became Knowledge, P. 271, Oxford University Press (2015).

26. J. M. Luttinger, On Negative Mass in the Theory of Gravitation, Gravity Research Foundation, (1951).

27. H. Bondi, Negative Mass in General Relativity, Reviews of Modern Physics. 29 (3): 423-428 (1957).

28. R. M. Price, Negative Mass Can be Positively Amusing, Am. J. Phys. 61(3): 216 (1993).

29. T. Pashby, Dirac's Prediction of the Positron: A Case Study for the Current Realism Debate, Perspectives on Science, 20, no. 4 p 440 (2012).

30. C. Anderson, The Positive Electron, Phys. Rev., 43, no. 6, pp. 491-494, Mar. 1933.

31. O. Igonkina, Inaugural Speech, Radboud University Facility Services, p. 9 (2015).

32. T. Zhang, Electric Charge as a Form of Imaginary Energy, Progress in Physics, 2 pp 79-83 (2008).

33. F. Soddy, The Complexity of Chemical Elements, The Scientific Monthly, 5, No. 5, pp. 451-462 (1917).

34. C.S. Unnikrishnan, and G. T. Gillies, The Electrical Neutrality of Atoms and of Bulk Matter, Metrologia, 41(5):S125 (2004).

35. I. Curie, and F. Joliot, $\beta$-Emission of Positive Electrons, C. R. Acad. Sci. 198, 254 (1934).

36. D. W. Engelkemeir, K. F. Flynn, and L. E., Glendenin, Positron Emission in the Decay of K40, Phys. Rev. 126 (5), 1818 (1962).

37. C.D. Simak (ed.), From Atom to Infinity, Readings in Modern Science, p.282, Minneapolis Star and Tribune Company, NY, (1964).

38. P. Blackett, Nobel Lectures, Physics 1942-1962, Elsevier Publishing Company, Amsterdam (1964).

39. J. H. Hubbell, Electron Positron Pair Production by Photons: A historical overview, Radiation Physics and Chemistry. 75 (6): 614-623 (2006).

40. S. P. Parker (ed.), Elementary Particles, McGraw-Hill Encyclopaedia of Science and Technology,' Fifth Edition, 5, McGraw-Hill Book Co., New York (1982).

41. J. Konya, and N. M. Nagy, Nuclear and Radio-chemistry, Elsevier, pp. 74-75, (2012).

42. S. Mertens, Direct Neutrino Mass Experiments, Journal of Physics: Conference Series. 718 (2): 022013 (2016).

43. P. G. Roll, R. Krotkove, and R. H. Dicke, The Equivalence of Inertial and Passive Gravitational Mass, Annals of Physics, 26, 442-517 (1964).

44. S. Carlip, Kinetic Energy and the Equivalence Principle, Am. J. Phys. 66, 409 (1998).

45. P.J. Mohr, et al. CODATA Recommended Values of the Fundamental Physical Constants, ARev. Mod. Phys, 84 1527-1605 (2012). 